\title{
Automatic Identification and Validation of Tie Points on Hyperspectral Satellite Images from CHRIS/PROBA
}

\author{
André R.S. Marçal \\ Faculdade de Ciências, Universidade do Porto \\ DMA, Rua do Campo Alegre, 687, 4169-007 Porto, Portugal
}

\begin{abstract}
There is great interest in the development of automatic geometric correction systems for satellite images. A fully automatic system, based exclusively on the identification of tie points (image to image control points) by image matching needs to use efficient selection and validation methods. Four Tie Point Suitability Indices (TPSI) are proposed to select the most suitable areas in an image to search for tie points. Three tie point validation parameters are also proposed. The validation parameters make use of the various spectral bands available in hyperspectral and multispectral satellite images. The proposed TPSIs and validation parameters were tested with hyperspectral high-resolution satellite images from the CHRIS/PROBA sensor.
\end{abstract}

\section{Introduction}

The manual registration and geometric correction of satellite images is a laborious and time-consuming task. The increasingly wider access to satellite images prompts an interest in the development of algorithms for the automatic or semiautomatic geometric correction of these images. As long as the images have certain similarities, it is possible to implement a fully automatic geometric correction system based on the identification of tie points (image to image control points) by image matching. The tie points are used to establish a transformation function between the input and the base or reference image, which once applied performs the geometric correction of the input image. In order for this process to be effective, a significant overlap (over 50\%) between the two images is required. However, even two images of the same place acquired by the same sensor, on different dates or with different viewing conditions, often look very different. This causes difficulties for a fully automatic geometric correction system based on automatic identification of tie points. One possible way to tackle this problem is to separate the image transformation process in two parts. Initially, a set of candidate tie points is searched for, but only a subset of trustable tie points are used to establish a first order (affine) transformation function. The second step consists of searching for a new set of tie points, but limiting the search to a small window centred on the locations predicted by the affine transformation. 
This methodology is strongly dependent on the ability to select the right candidate tie points that are used to establish the affine transformation function. The purpose of this work is to propose a methodology to select and validate tie points identified on hyperspectral satellite images. The method was developed for hyperspectral satellite images from CHRIS/PROBA.

CHRIS/PROBA is the first hyperspectral satellite sensor with pointing capabilities and high spatial resolution 11. A CHRIS/PROBA scene is composed of 5 images with different viewing angles, with fly-by Zenith Angles (ZA) of 55, 36, 0, -36 and 36 degrees [2]. In the most common operational mode, the sensor acquires data with a nominal spatial resolution of 17 meters over the full swath $(13 \mathrm{~km})$, with 18 spectral bands from 400 to $1050 \mathrm{~nm}[3$.

\section{Method}

In this section a method to establish a set of candidate tie points is described, and a number of parameters that can be used for a validation criteria proposed.

\subsection{Tie Point Selection by Image Matching}

The tie point selection process is based on image matching by normalized twodimensional cross-correlation in the spatial domain. A target matrix $T$ (of size $t$, usually small) is established in the reference image and a search window $S$ (of size $s$, larger than $t$ ) is examined in the input image. The convolution between $T$ and all sub-window of $S$ (of size $t$ ) is performed, resulting is a set of correlation coefficients, from -1 to 1 . The best match will be the pixel of highest correlation in the search window. The MATLAB implementation of the normalized twodimensional cross-correlation function was used in this work [4.

\subsection{Tie Point Suitability Indices}

The purpose of a Tie Point Suitability Index (TPSI) is to identify the best locations on an image to search for tie points. These locations should have distinct features in order to maximise the chances of a correct selection on the image matching process. For a given target matrix of size $t$ (for example $3 \times 3$ for $t=3)$ a TPSI value is attributed to each pixel of the image, thus producing a TPSI image, where the highest values should correspond to the most promising locations to search for tie points. Four TPSI are proposed: Basic $(B)$, Composed $(C)$, Ratio $(R)$ and Prewitt $(P)$.

For a sub-section of an image of size $\mathrm{t}$ ( $\mathrm{t}$ by $\mathrm{t}$ pixels), $H h$ is the highest possible sum of pixel values, or Digital Numbers (DNs), from two horizontally adjacent pixels, $H v$ is the highest sum of DNs from two vertically adjacent pixels, and $L h$ and $L v$ are the lowest DN sums for two pixels horizontally and vertically adjacent. For example, the value of $H h$ of Figure 1]s section (a) is 0.74, as the highest pair is formed by the pixels with DN values of 0.36 and 0.38 on the top right corner. The values for the other parameters for this section are: $L h=0.51$, 


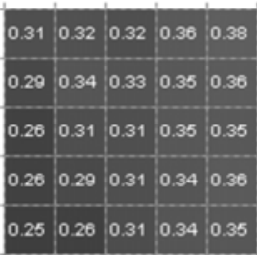

(घ)

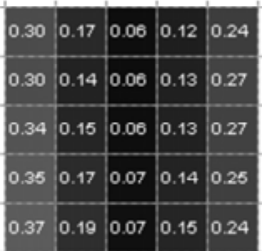

(b)

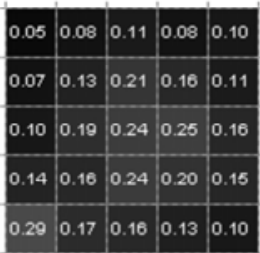

(c)

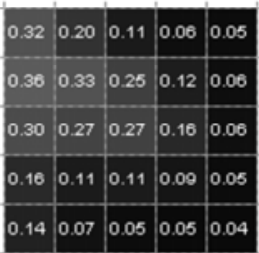

(d)

Fig. 1. Example of $5 \times 5$ test sites

Table 1. TPSI values for the $5 \times 5$ test sites of figure 1 (band 14 as reference)

\begin{tabular}{c|cccc|c|c|c|c} 
Site & Hh & Lh & Hv & Lv & Basic TPSI & Composed TPSI & Ratio TPSI & Prewitt TPSI \\
\hline A & 0.74 & 0.51 & 0.74 & 0.51 & 0.1903 & 0.0349 & 0.0057 & 0.0086 \\
B & 0.56 & 0.18 & 0.72 & 0.12 & 0.7188 & 0.3605 & 0.0420 & 0.0028 \\
C & 0.49 & 0.13 & 0.48 & 0.12 & 0.6028 & 0.3539 & 0.1122 & 0.0117 \\
D & 0.69 & 0.09 & 0.68 & 0.09 & 0.7710 & 0.5849 & 0.1791 & 0.1311 \\
\hline
\end{tabular}

$H v=0.74$ and $L v=0.51$. The values for the other 3 sites of Figure 1 are presented in Table 1.

The Basic TPSI $(B)$ uses the highest and lowest pairs, regardless of the orientation, to compute an index from 0 to 1 , using (1), where $H=\operatorname{Max}\{H h, H v\}$ and $L=\operatorname{Max}\{L h, L v\}$. The Basic TPSI $(B)$ has high values when the difference between the highest DN pair and lowest DN pair is high. In homogeneous areas the values of $B$ will be low. The Composed TPSI $(C)$, computed by (2), has some similarities with the Basic TPSI. However, the information about horizontal and vertical variability within the matrix is used separately. This index only reaches high values when there is a large difference between the highest and lowest DN pairs both horizontally and vertically.

$$
\begin{gathered}
B=\frac{H-L}{H+L} \\
C=\frac{(H v-L v) \times(H h-L h)}{(H v+L v) \times(H h+L h)}
\end{gathered}
$$

The Ratio TPSI $(R)$ is computed in a different way from $B$ and $C$, searching for edges locally. For each pair of horizontally adjacent pixels in the sub-section of the image being tested, the ratio between the absolute difference and the sum of their DNs is calculated. The maximum for all pairs is selected as $R h$. The same process is done for all vertical pairs, resulting in the maximum ratio $R v$. The Ratio TPSI is obtained by multiplying $R h$ and $R v$. The index only has high values when there are both strong horizontal and vertical edges.

The final TPSI proposed is based on a standard edge detector - the Prewitt operator [6]. The Prewitt TPSI is obtained by the multiplication of the results of $3 \times 3$ horizontal and vertical Prewitt operators applied to the input image. 
Again, this index will only reach high values when there are strong edges, both horizontally and vertically, within the image sub-section tested.

As an illustration, four $5 \times 5$ sections of an image are presented in Figure 1 . These are 5 by 5 pixel sections used to compute the TPSI for the central pixel $(t=5)$. The examples in Figure 1 illustrate different cases: (a) a homogeneous region, (b) a strong vertical edge, (c) a weak and small peak, and (d) an area with two clearly distinct zones, providing both horizontal and vertical edges. The site (d) should definitely be the best choice for a candidate tie point and the second best should be site (c). Sites (a) and (b) are clearly inadequate to find a tie point. The values of the TPSIs for these four image sections are presented in Table 1. All indices rated site (d) as the best choice, but the TPSIs $B$ and $C$ failed to identify site (c) as the second best choice. These two indices ranked highly the strong vertical edge in site (b).

\subsection{Tie Point Validation}

Initially a spectral band is used to produce a TPSI. A criterion will select which pixels will be used as candidate tie points. For example, the image can be divided into sectors, and the pixel with highest TPSI in each sector selected.

For each pixel selected as candidate tie point in the base image, the matching process will provide a conjugate pair in the input image. This will be the location in the search window where the convolution between the target window and the search sub-window is maximum. However, this will not necessarily be a suitable match, as the presence of clouds, noise, or other similar locations elsewhere might result on the selection of the wrong location. It is important to have a consistent criterion to reject these bad matches. The hyperspectral characteristic of the images can provide additional information to properly identify the correct matches.

A convolution between the target window and the search sub-window, centred on the location selected by the image matching process, is performed in several spectral bands. In this work only 9 bands were used (bands 1, 4, 6, 8, 10, 12, 14, 16 and 18) but more bands could be easily used. Three parameters are considered: (i) the number of bands with a correlation coefficient $(r)$ above $0.95(N 1)$, (ii) the number of bands with $r>0.90(N 2)$ and (iii) the average of the 3 highest $r$ values $(R 123)$. An adequate match will hopefully score high in all 3 parameters while a wrong match should score low in at least one of them.

\section{$3 \quad$ Results}

Three CHRIS/PROBA scenes of the same location were acquired to test the performance of the TPSIs and the validation parameters. The image centre target was a point in Arcos de Valdevez, northwest Portugal, with longitude -8.42 and latitude 41.8. Each image scene includes 5 images (766 by 748 pixels) with different viewing angles, each with 18 spectral bands. Due to the uncertainty in CHRIS/PROBA pointing, the centre might be displaced by as much as 
$7 \mathrm{~km}$, which is more than half the image size (image swath of $13 \mathrm{~km}$ ) [1]. This is true both for images acquired on different dates, and for different viewing angle images of the same scene.

\subsection{Testing Strategy}

Five pairs of CHRIS/PROBA images were selected for testing. Table 2 indicates the main characteristics of the images used (ZA-Zenith Angle). The image pairs tested were the pairs formed by the three vertical views (IM1 to IM2, IM1 to IM3 and IM2 to IM3), and the pairs formed between the vertical view and two oblique views for image 1 (IM1 to IM1B and IM1 to IM1C). Figure 2 shows an example of a vertical view image (IM3), the near infrared band $14(781 \mathrm{~nm})$ [2]. In this image it is easy to distinguish the noticeable feature of the river that crosses the image nearly horizontally, which given its irregularity should provide good locations to search for tie points.

Table 2. CHRIS/PROBA images used in the TPSI test (ZA-Zenith Angle)

\begin{tabular}{crrrr}
\hline LABEL & Aquisition DATE & Fly-By ZA & ObSERvation ZA & Solar ZA \\
\hline IM1 & 11042006 & 0 & 4.7 & 35.0 \\
IM1B & 11042006 & +36 & 28.3 & 35.0 \\
IM1C & 11042006 & -36 & 33.4 & 35.0 \\
IM2 & 28052006 & 0 & 3.6 & 30.0 \\
IM3 & 24052006 & 0 & 6.2 & 23.0 \\
\hline
\end{tabular}

For each pair of images, one was selected as base and the other as input image. Between 9 and 15 control points were identified manually for each image pair, which were used to establish first order and second order polynomial transformation functions. The TPSIs B, C, R and P were computed for selected reference bands of the base images. Only bands 1, 6, 10, 14 and 18 were used as reference. As an example, Figure 2 (centre) shows the Composed TPSI (C) produced for IM3 with reference band 14 (the image displayed is $C \times 2$ as the original image has low contrast). The next step is to select a reasonable number of widespread tie point candidates. This goal is achieved by establishing a 5 by 5 grid of nonadjacent sectors. An illustration of this grid is displayed over a TPSI image in Figure 2 (right). For each TPSI image, the pixel with maximum value on each sector is selected as a candidate tie point. The image-matching algorithm is used to select the conjugate pixel on the input image, with a search window size of 251 by 251 pixels. The central pixel of the sub-window with maximum correlation coefficient is selected as the tie point conjugate pair. The correlation between the base and input image for a $5 \times 5$ window is computed for this pair for all 9 bands. The 3 validation parameters $(N 1, N 2$ and $R 123)$ are also computed.

A tie point provided by the image matching process is evaluated according to the root mean square difference between its coordinates in the input image and those predicted by the second order polynomial transformation function 

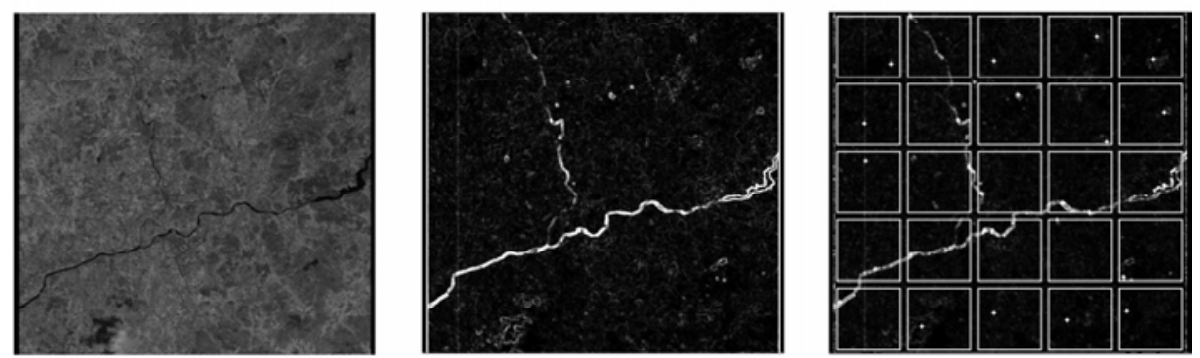

Fig. 2. IM3 Band 14 (left), Composed TPSI (centre), Ratio TPSI with sectors and points selected (right)

established for the image pair. If the difference was less than 2 pixels the point was labelled as correct, if it was above 5 pixels it was labelled as incorrect, and those pixels with a difference between 2 and 5 pixels were labelled as doubtful. The pixels that the polynomial transformation function projected to a location outside the input image were considered invalid, as no successful matching was possible. Those points were discarded from subsequent analysis.

\subsection{Reference Band Test}

The first test was to evaluate the importance of the spectral band used as reference. The procedure described in the previous section was applied to the image pair IM1 (base) and IM2 (input) using bands 1, 6, 10, 14 and 18 as reference bands. The results are summarised in Table 3 Only the correct (good) and the incorrect (bad) matches are displayed in the table. For each of the four TPSI the variables tested were the number of tie points, and the validation parameters $N 1, N 2$ and $R 123$. Ideally one would have a large number of correct tie points and few incorrect tie points. The validation criteria should have high values for correct points and low values for incorrect points. The values underlined in Table 3 correspond to the best reference band for each TPSI, and in bold is the best overall for each parameter tested. For example, the best combination in terms of the number of valid (good) tie points was to use the Ratio TPSI with band 10 as reference. This combination resulted in 16 correct and only 5 incorrect tie points ( 2 were doubtful and the remaining 2 invalid).

The best scenario for the validation parameters is to have a large difference between the values of good and bad tie points. This is a requirement to make the parameter able to properly discriminate the good and bad (valid or invalid) tie points. The values underlined in Table 3 correspond to the reference bands that proved more efficient in this context. The Ratio TPSI performed best in all 4 parameters tested. The reference band 14 seems to be the most efficient in terms of tie point validation, while band 10 or band 1 are good choices in terms of getting a high number of valid tie points. This might be partially justified by the spectral location of these bands. Band 1 is in the blue part of the visible spectrum (442 nm), Band 10 in the red edge $(703 \mathrm{~nm})$ and Band 14 in the near infrared 
Table 3. Summary of the TPSI performance using 5 different reference bands (image pair IM1-IM2)

\begin{tabular}{|c|c|c|c|c|c|c|c|c|}
\hline \multirow{2}{*}{$\begin{array}{l}\text { TPSI: } \\
\text { REFERENCE / TP type }\end{array}$} & \multicolumn{2}{|c|}{ BASIC } & \multicolumn{2}{|c|}{ COMPOSED } & \multicolumn{2}{|c|}{ RATIO } & \multicolumn{2}{|c|}{ PREWITT } \\
\hline & Good & Bad & Good & Bad & Good & Bad & Good & Bad \\
\hline No. TPs & & & & & & & & \\
\hline BAND 1 & $\underline{14}$ & $\underline{7}$ & $\underline{14}$ & $\underline{7}$ & 14 & 7 & $\underline{12}$ & $\underline{9}$ \\
\hline BAND 6 & $\overline{10}$ & $\overline{10}$ & $\overline{9}$ & $\overline{10}$ & 10 & 11 & $\overline{10}$ & $\overline{12}$ \\
\hline BAND 10 & 11 & 8 & 11 & 8 & $\underline{16}$ & $\underline{\mathbf{5}}$ & 8 & 10 \\
\hline BAND 14 & 11 & 7 & 9 & 9 & $\overline{10}$ & $\overline{6}$ & 8 & 10 \\
\hline BAND 18 & 8 & 11 & 12 & 6 & 9 & 8 & 11 & 8 \\
\hline N1 & & & & & & & & \\
\hline BAND 1 & 5.00 & 2.21 & 5.71 & 2.86 & 5.57 & 0.93 & 4.33 & 0.83 \\
\hline BAND 6 & 6.80 & 2.50 & $\underline{6.67}$ & $\underline{2.30}$ & 3.40 & 1.73 & 5.90 & 1.42 \\
\hline BAND 10 & $\underline{6.55}$ & $\underline{1.88}$ & 5.73 & 2.25 & 4.13 & 0.80 & $\underline{6.00}$ & $\underline{0.50}$ \\
\hline BAND 14 & $\overline{5.45}$ & $\overline{4.00}$ & 5.56 & 4.00 & 6.50 & $\underline{0.50}$ & $\overline{5.38}$ & $\overline{3.10}$ \\
\hline BAND 18 & 6.25 & 3.18 & 6.42 & 2.67 & $\overline{6.22}$ & $\overline{0.88}$ & 5.73 & 2.50 \\
\hline N2 & & & & & & & & \\
\hline BAND 1 & 6.57 & 4.07 & 7.14 & 4.43 & 7.57 & 2.57 & 7.67 & 3.25 \\
\hline BAND 6 & 7.70 & 4.60 & 7.89 & 4.40 & 6.90 & 2.55 & 7.10 & 3.17 \\
\hline BAND 10 & $\underline{7.64}$ & $\underline{4.38}$ & $\underline{7.55}$ & $\underline{3.25}$ & 7.06 & 3.20 & $\underline{8.00}$ & $\underline{2.90}$ \\
\hline BAND 14 & $\overline{6.91}$ & $\overline{6.00}$ & $\overline{7.11}$ & $\overline{5.56}$ & 7.90 & $\underline{2.00}$ & $\overline{6.50}$ & $\overline{4.40}$ \\
\hline BAND 18 & 7.75 & 5.27 & 7.42 & 5.17 & $\overline{7.56}$ & $\overline{2.63}$ & 6.55 & 4.25 \\
\hline R123 & & & & & & & & \\
\hline BAND 1 & 0.973 & 0.947 & 0.976 & 0.955 & 0.968 & 0.907 & 0.963 & 0.925 \\
\hline BAND 6 & 0.978 & 0.964 & 0.981 & 0.954 & 0.958 & 0.923 & 0.972 & 0.918 \\
\hline BAND 10 & 0.978 & 0.944 & 0.971 & 0.923 & 0.960 & 0.848 & 0.974 & 0.890 \\
\hline BAND 14 & 0.921 & 0.821 & $\underline{0.946}$ & $\underline{0.663}$ & 0.951 & 0.357 & $\underline{0.922}$ & $\underline{0.640}$ \\
\hline BAND 18 & $\underline{0.963}$ & $\underline{0.838}$ & $\overline{0.965}$ & $\overline{0.755}$ & $\overline{0.946}$ & $\overline{0.480}$ & $\overline{0.929}$ & $\overline{0.769}$ \\
\hline
\end{tabular}

(781nm) 2]. There is considerable scattering by the Earth's atmosphere for low visible wavelengths (particularly blue), which tends to reduce the variability of the surface reflectances in Band 1. On the contrary, the spectral signatures of water and vegetation are clearly distinguishable in the near infrared. However, using near infrared reference bands, it is very likely that some vegetation features are selected as candidate tie points, which will often fail to produce a suitable match due to vegetation change between the two image acquisition dates.

Overall, the performance of band 10 as reference band can be considered rather good, except for the validation parameter $R 123$, which has a small difference between good and bad tie points.

\subsection{TPSI Test}

Another test of the TPSI performance was done using the 5 image pairs available. The reference band used was band 10, as this seems to offer a good compromise between the various parameters of interest. The results are summarised in Table 4, again with only the correct (good) and incorrect (bad) tie points 
Table 4. Summary of the TPSI performance using 5 images pairs (reference band 10)

\begin{tabular}{cccccc|cc|cc}
\hline & TPSI: & \multicolumn{2}{c}{ BAsIC } & \multicolumn{3}{c}{ Composed } & \multicolumn{2}{c}{ RATIO } & \multicolumn{2}{c}{ PREWITT } \\
IMAGE PAIR / & TP type: Good & Bad & Good & Bad & Good & Bad & Good & Bad \\
\hline No. TPs & & & & & & & & \\
IM1 TO IM2 & 11 & 8 & 11 & 8 & $\underline{\mathbf{1 6}}$ & $\underline{\mathbf{5}}$ & 8 & 10 \\
IM1 TO IM3 & $\underline{8}$ & $\underline{8}$ & 7 & 7 & 5 & 12 & 4 & 13 \\
IM2 TO IM3 & 12 & 8 & 12 & 8 & $\underline{13}$ & $\underline{7}$ & 10 & 11 \\
IM1 TO IM1B & $\underline{7}$ & $\underline{14}$ & 7 & 15 & 6 & 19 & 4 & 19 \\
IM1 TO IM1C & 6 & 16 & 5 & 18 & $\underline{7}$ & $\underline{17}$ & 6 & 17 \\
\hline N1 & & & & & & & & \\
IM1 TO IM2 & 6.5 & 1.9 & 5.7 & 2.3 & 4.1 & 0.8 & $\underline{6.0}$ & $\underline{0.5}$ \\
IM1 TO IM3 & 6.0 & 2.3 & 5.7 & 2.4 & 5.8 & 0.9 & $\underline{\mathbf{6 . 8}}$ & $\underline{\mathbf{1 . 2}}$ \\
IM2 TO IM3 & 7.5 & 3.0 & $\underline{7.7}$ & $\underline{3.1}$ & 5.5 & 1.4 & 5.8 & 1.5 \\
IM1 TO IM1B & 7.7 & 2.7 & $\underline{7.9}$ & $\underline{2.7}$ & 7.3 & 2.7 & 6.0 & 2.2 \\
IM1 TO IM1C & 7.7 & 4.3 & 6.6 & 3.8 & 3.4 & 1.5 & $\underline{5.2}$ & $\underline{1.6}$ \\
\hline N2 & & & & & & & & \\
IM1 TO IM2 & 7.6 & 4.4 & 7.5 & 3.3 & 7.1 & 3.2 & $\underline{\mathbf{8 . 0}}$ & $\underline{\mathbf{2 . 9}}$ \\
IM1 TO IM3 & 7.4 & 3.6 & 7.1 & 3.9 & $\underline{6.8}$ & $\underline{2.5}$ & 7.3 & 3.3 \\
IM2 TO IM3 & 8.7 & 5.0 & 8.7 & 4.4 & 7.8 & 4.0 & $\underline{7.5}$ & $\underline{3.1}$ \\
IM1 TO IM1B & 8.9 & 4.9 & $\underline{8.9}$ & $\underline{4.4}$ & 8.5 & 4.3 & 8.3 & 4.0 \\
IM1 TO IM1C & 8.0 & 6.0 & 7.8 & 5.6 & $\underline{7.4}$ & $\underline{3.5}$ & 7.2 & 4.4 \\
\hline R123 & & & & & & & & \\
IM1 TO IM2 & 0.978 & 0.944 & 0.971 & 0.923 & $\underline{\mathbf{0 . 9 6 0}}$ & $\underline{\mathbf{0 . 8 4 8}}$ & 0.974 & 0.890 \\
IM1 TO IM3 & $\underline{0.980}$ & $\underline{0.908}$ & 0.980 & 0.932 & 0.973 & 0.903 & 0.987 & 0.924 \\
IM2 TO IM3 & 0.981 & 0.957 & 0.981 & 0.940 & 0.970 & 0.923 & $\underline{0.975}$ & $\underline{0.912}$ \\
IM1 TO IM1B & 0.985 & 0.911 & $\underline{0.986}$ & $\underline{0.904}$ & 0.969 & 0.908 & 0.972 & 0.929 \\
IM1 TO IM1C & 0.982 & 0.944 & 0.982 & 0.928 & $\underline{0.941}$ & $\underline{0.882}$ & 0.969 & 0.918 \\
\hline
\end{tabular}

displayed. The underlined values in this Table correspond to the best TPSI performer, for each image pair and parameter. The bold indicates the best performance overall for a parameter. Out of the 20 image pairs and parameters tested, the number of wins was 3 for the Basic TPSI $(B), 4$ for $C, 7$ for $R$ and 6 for $P$. The $R$ and $P$ indices got 2 best overall rates each. Although the results do not seem to indicate a clear favourite, the Ratio and Prewitt indices performed better.

The results in Tables 3 and 4 suggest that a suitable discrimination criteria based on the $N 2$ value can be established. The results seem to indicate that a threshold of 5 could perhaps be effective to distinguish between good and bad tie points. However, the values presented in these tables are average values, calculated for all tie point candidates labelled as good or bad. A detailed analysis was carried out to investigate if this could be an appropriate discrimination criterion. The results are summarised in Table 5. The accuracy of this criterion depends on the TPSI index, reference band and the limiting threshold (l) considered, but are generally around $80 \%$. 
Table 5. Evaluation of a discriminative criteria based on $N 2$

\begin{tabular}{|c|c|c|c|c|}
\hline LABEL & RATIO 10 & PREWITT 10 & RATIO 14 & PREWITT 14 \\
\hline No. Points & 107 & 101 & 104 & 100 \\
\hline Success rate $(l=6)$ & $79.1 \%$ & $84.1 \%$ & $83.9 \%$ & $76.5 \%$ \\
\hline Success rate $(l=5)$ & $78.1 \%$ & $80.4 \%$ & $81.0 \%$ & $74.2 \%$ \\
\hline
\end{tabular}

\section{Conclusions}

The successful implementation of a fully automatic geometric correction system, based on the identification of tie points (image to image control points) by image matching, is dependent both on the ability to identify suitable areas to search for tie points, and to validate the candidate tie points. Four Tie Point Suitability Indices (TPSI) were proposed, which aim to select the most suitable areas to be used as candidate tie points in an image. Three tie point validation parameters were also proposed, which can be used with hyperspectral or multispectral images. The validation parameters make use of the fact that there is a high correlation between the neighbourhoods of correctly matched tie point for a large number of spectral bands. The proposed TPSIs and validation parameters were tested with 5 CHRIS/PROBA hyperspectral high-resolution satellite images. A criterion to distinguish between correct and incorrect candidate tie points was tested, with an accuracy of about $80 \%$. The results are promising but further research is still required in order to establish the most effective TPSI and validation criteria.

\section{Acknoledgments}

This work was done with the support of Centro de Investigação em Ciências Geo-Espaciais, Faculdade de Ciências da Universidade do Porto, financed by the Portuguese National Science Foundation (Fundação para a Ciência e a Tecnologia - FCT).

\section{References}

1. Alonso, L., Moreno, J.: Advances and limitations in a parametric geometric correction of CHRIS/PROBA data. In: Proc. of the 3rd ESA CHRIS/Proba Workshop, March 21-23, ESRIN, Frascati, Italy ESA SP-593 (2005)

2. Cutter M. A.: CHRIS data format, revision 4.2 SIRA, Kent, UK (2005)

3. Barnsley, M.J., Settle, J.J., Cuter, M.A., Lobb, D.R., Teston, F.: The PROBA/CHRIS Mission: A Low-Cost Smallsat for Hyperspectral Multiangle Observations of the Earth Surface and Atmosphere. IEEE Transactions on Geoscience and Remote Sensing 45, 1512-1520 (2004)

4. Using Matlab, Version 6.5. The MathWorks, Inc. Natick. MA (2002)

5. Gonzalez, R.C., Woods, R.E.: Digital Image Processing. Prentice-Hall, Englewood Cliffs (2002)

6. Gonzalez, R.C., Woods, R.E., Eddins, S.L.: Digital Image Processing using MATLAB. Prentice-Hall, Englewood Cliffs (2004) 